\title{
APPROCHE ÉDUCATIVE DE LA SIMULATION DES MICROSYSTÈMES DANS LEUR ENVIRONNEMENT
}

\author{
Bruno ESTIBALS ${ }^{1,2}$, Corinne ALONSO ${ }^{2,3}$, Jean-Yves FOURNIOLS ${ }^{1,2}$, \\ Jean-Louis NOULLET ${ }^{1}$, Christophe VIEU ${ }^{1,2}$, Henri CAMON ${ }^{2}$, Alain CAZARRÉ ${ }^{2,3}$, \\ Augustin MARTINEZ ${ }^{1,2}$ \\ ${ }^{1}$ Institut National des Sciences Appliquées de Toulouse (INSAT) \\ Département de Génie Electrique et d’Informatique \\ 135, avenue de Rangueil - F-31077 Toulouse Cedex 04 \\ ${ }^{2}$ Laboratoire d'Analyse et d'Architecture des Systèmes (LAAS-CNRS) \\ 7, avenue du Colonel Roche - F-31077 Toulouse Cedex 04 \\ Tél.: 0561336443 - Télécopie : 0561336942 - E-Mail : bruno.estibals@laas.fr \\ ${ }^{3}$ Université Paul Sabatier - Toulouse III \\ Département d'Electronique, Electrotechnique et d'Automatique (EEA) \\ 118, Route de Narbonne - F-31062 Toulouse Cedex 4
}

Résumé : cet article a pour but de présenter une approche éducative de la simulation d'un microsystème à travers un micro-miroir et de son alimentation, à l'aide de deux outils, COVENTOR et SABER. Une confrontation des résultats obtenus à l'aide des simulateurs est effectuée avec un modèle analytique.

\section{INTRODUCTION.}

Le concept de microsystèmes ou MEMS (Micro Electro Mechanical Systems), né dans les années 80 , est basé sur la cohabitation de structures à fonctions mécaniques et électriques de très faibles dimensions sur une même puce[1-2]. Ces réalisations, d'une très grande variété, sont rendues possibles grâce à l'utilisation des techniques classiques de la microélectronique, telles que la photolithographie, les techniques de dépôts, de gravure, de report, d'interconnexion, et d'assemblage [3]. Ces dernières permettent ainsi l'émergence de nombreux produits sur des domaines d'application de plus en plus vastes. Les micro-capteurs, première application des MEMS, envahirent par exemple rapidement les secteurs de l'automobile, permettant ainsi l'introduction de coussins gonflables de sécurité (airbags) grâce aux accéléromètres micro-usinés, ou du biomédical avec des micro-capteurs à usage unique pour la mesure de pression artérielle. De nouveaux produits sont aujourd'hui en développement et verront bientôt le jour industriellement tels des micro-pompes pour l'injection de médicaments, des commutateurs Radio Fréquence pour les télécommunications mobiles et bien d'autres encore [4].

Concevoir des microsystèmes implique des étapes de Conception, simulation et de fabrication de systèmes de taille très réduites. Ils sont créés en combinant des fonctions individuelles sur la même puce, qui, associées entre elles, assureront une fonction globale. Ces remarques, quoique généralistes, montrent 
bien le caractère pluri-disciplinaire que doivent posséder les concepteurs de microsystèmes pour réaliser de tels composants: mécanicien, électro-mécanicien, ingénieur matériau, électronicien, etc... La diversité des domaines techniques abordés nécessite l'utilisation d'outils d'aide à la conception, permettant de réaliser au plus près de la réalité des simulations et d'éviter ainsi un nombre important d'essais technologiques, fortement coûteux.

Les départements de Génie Electrique et Informatique (DGEI) et de Génie Physique (DGP) de l'INSA de Toulouse ont associé leurs compétences pour former, depuis la rentrée de Septembre 2000, des ingénieurs multidisciplinaires, préparés à concevoir et à réaliser des microsystèmes [5]. Une première expérience, montrant la conception et la réalisation d'un actionneur thermique, avait déjà été présentée[6].

Pour illustrer les nouveaux besoins d'intégration de la source d'énergie avec son application, nous présentons dans cet article la simulation d'un micro-miroir dans son environnement de commande. Après une description de la structure et des principales équations régissant son fonctionnement, nous effectuerons, dans un premier temps, des simulations en statique, afin d'extraire les valeurs des tensions d'actionnement. Nous utiliserons pour cela le logiciel COVENTORWARE développé par COVENTOR [7]. Un modèle électrique du miroir est ensuite extrait grâce à COVENTOR, puis introduit sous SABER [8], afin d'effectuer la simulation du miroir avec son alimentation.

\section{SIMULATIONS AUX ÉLÉMENTS FINIS.}

\subsection{Présentation de la structure.}

Les microsystèmes optiques ou MOEMS (Micro Optical Electro Mechanical Systems) apparaissent actuellement être les seuls à pouvoir assurer toute une série de fonctions indispensables aux réseaux optiques : atténuation, insertion/extraction de canaux et connexion, commutation, en traitant directement le signal lumineux. Cette approche est aujourd'hui une alternative à de nombreuses et coûteuses transitions optoélectroniques et électro-optiques, qui sont une limite en débit de transmission [9].

Les micro-miroirs micro-usinés sur silicium peuvent être considérés comme un élément central des microsystèmes optiques. Ils peuvent en effet être utilisés :

- Pour rediriger des faisceaux : ils doivent alors garantir un certain nombre de positions stables, afin d'aiguiller une longueur d'onde d'une fibre optique d'entrée vers une fibre de sortie bien déterminée. C'est le cas du DWDM (Dense Wavelength Division Multiplexing).

- Pour effectuer de l'optique adaptative : c'est une technique dont le but consiste à restaurer en temps réel la qualité des images détériorées par un phénomène. Elle consiste à utiliser des miroirs à membrane déformante pour redresser des fronts d'ondes, et restituer un front d'onde le plus conforme possible à l'original. 


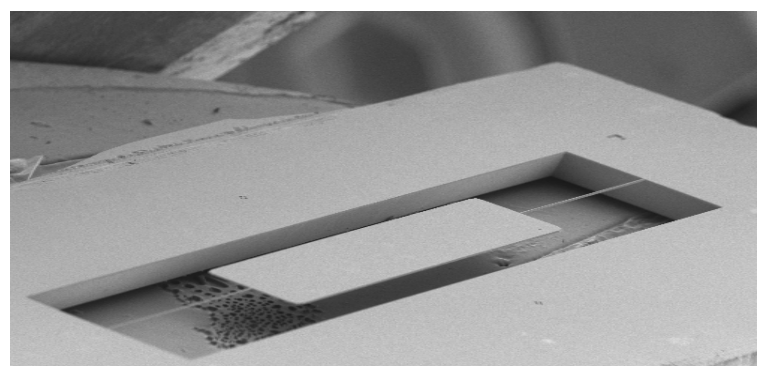

a.

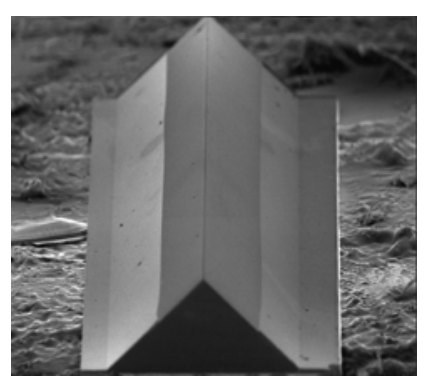

b.

Figure 1. Schéma d’un micro-miroir.

a. Miroir - b. Partie actionnement, positionnée sous le miroir.

La figure 1 présente un exemple de structure à micro-miroir, dans laquelle le substrat a été supprimé pour plus de simplicité. Celle-ci comprend :

- La plaque de réflexion, qui consiste en une plaque de silicium suspendue par deux barres de torsion. L’ensemble est micro-usinée sur silicium.

- La partie actionnement, qui utilise ici un actionnement électrostatique. Pour cela, deux électrodes de commande sont placées sous le miroir.

L'application d'une tension sur une des électrodes crée des forces électrostatiques engendrant la rotation du miroir autour de son axe. On considèrera par la suite que les forces électrostatiques créent un moment électrostatique $\vec{M}_{e}$. Les différents couples s'opposant au mouvement du miroir sont :

- Le moment d'inertie : proportionnel à l'accélération, dépendant du moment d'inertie $I$ de la plaque et de l'angle de rotation $\theta: I . \ddot{\theta}$,

- Le couple mécanique : il dépend de la constante de raideur en torsion $k$ de la barre, et est fonction de l'angle de rotation $\theta: k . \theta$,

- L'amortissement : considéré comme proportionnel à la vitesse angulaire. En première approximation, comme les angles de variation sont faibles, ces effets sont considérés comme négligeable.

De ce fait, en considérant que le moment d'inertie est égal à la somme des moments des forces extérieures s'exerçant sur le système, l'équation du mouvement se déduit en projetant l'équation vectorielle sur l'axe de rotation :

$$
\vec{M}_{\text {Mécanique }}+\vec{M}_{e}+\vec{M}_{\text {Inertie }}=0
$$

Le facteur 2 dépend du fait que la plaque réfléchissante est suspendue par deux barres de torsion.

\subsection{Etude théorique.}

\subsubsection{Calcul de la raideur de la barre de torsion.}

La première partie consiste à calculer la courbe d'actionnement statique du micro-miroir en implémentant, sous MATLAB par exemple, les équations donnant la tension d'actionnement statique $V$ en fonction de l'angle de rotation $\theta$. 
La première donnée à évaluer est la raideur de la barre de torsion $k$. Fonction de ses dimensions géométriques, elle influe en effet sur la valeur de la tension d'actionnement $V$ qui sera appliquée sur l'une ou l'autre des électrodes. La raideur est donnée par [10]:

$$
k=\frac{G}{l} \cdot a b^{3} \cdot\left[\frac{16}{3}-3 \cdot 36 \cdot \frac{b}{a} \cdot\left(1-\frac{b^{4}}{12 \cdot a^{4}}\right)\right] \quad \text { pour } a \geq b
$$

où $a, b$ et $l$ sont les dimensions de la section de la barre de torsion et $G$ le module de rigidité (ou de Coulomb) du matériau utilisé pour réaliser la barre. Une fois calculée, on cherche à évaluer la valeur de la tension d'actionnement $V$.

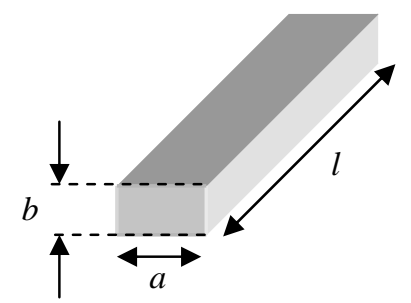

Figure 2. Schéma d’un micro-miroir.

Si l'on prend l'exemple du ressort étudié par la suite, la raideur de la barre de torsion de largeur $b$ $10 \mu \mathrm{m}$, d'épaisseur $a 15 \mu \mathrm{m}$ et de longueur $l 600 \mu \mathrm{m}$ est égale à :

$k=\frac{50850 \cdot 10^{6}}{600 \cdot 10^{-6}} \cdot 15 \cdot 10^{-6} \times\left(10 \cdot 10^{-6}\right)^{3} \cdot\left[\frac{16}{3}-3 \cdot 36 \cdot \frac{10}{15} \cdot\left(1-\frac{10^{4}}{12 \times 15^{4}}\right)\right] \Leftrightarrow k=3 \cdot 98 \cdot 10^{-7} \mathrm{~N} \cdot \mathrm{m}$

\subsubsection{Calcul de la tension d'actionnement : équations de base.}

La théorie de l'électrostatique est décrite par les équations de Maxwell pour le champ électrostatique, à savoir par :

$$
\begin{aligned}
& \overrightarrow{\operatorname{rot}} E=-\frac{\partial \vec{B}}{\partial t}=0 \\
& \operatorname{div} \vec{E}=\frac{\rho}{\varepsilon_{0}}
\end{aligned}
$$

Dans ce cas, $\vec{E}$ et $\vec{B}$ sont les vecteurs champ électrique et magnétique, $\rho$ la densité de charge d'espace et $\varepsilon_{0}$ la permittivité du milieu ambiant. Si l'on considère que le champ magnétique ne dépend pas du temps, le terme $\overrightarrow{r o t} E$ est nul. On peut donc définir le potentiel scalaire électrostatique $V$ par :

$$
\vec{E}=-\overrightarrow{\operatorname{grad}} V
$$

La distribution du champ électrostatique $V$ décrit complètement la situation électrostatique du domaine. Cette distribution est obtenue en associant les équations (3) et (4) :

$$
\Delta V=\frac{\rho}{\varepsilon_{0}}
$$

Cette expression est une équation aux dérivées partielles. Si la charge d'espace est égale à zéro sur le domaine entier, l'équation homogène ainsi obtenue s'écrit par :

$$
\Delta V=0
$$


L'analyse électrostatique correspond à la résolution de cette dernière équation sur la géométrie du système et pour les conditions aux limites considérées. De cette analyse, toutes les quantités électrostatiques peuvent être déduites : champ électrostatique $\vec{E}$, densité de charge $\sigma$. Cette répartition de charge entraîne une pression électrostatique $\vec{\Pi}$, normale à la surface du conducteur, donnée par :

$$
\vec{\Pi}=\frac{\sigma^{2}}{2 \varepsilon_{0}} \cdot \vec{n}
$$

\subsubsection{Calcul de la tension d'actionnement : expression du moment.}

La répartition du potentiel électrostatique est l'élément fondamental dans le calcul de l'interaction électrostatique et dépend fortement de la géométrie de l'espace entre les électrodes et le miroir. Considérons ici la géométrie la plus utilisée dans la littérature : la configuration «électrodes planes ». Deux approximations sont à faire dans ce cas :

- pour de petits angles, on néglige le non-parrallélisme des plaques dans le calcul du champ électrostatique,

- pour de petits angles, on néglige le gap entre les électrodes et le miroir devant la longueur du miroir.

Ainsi, les lignes équipotentielles sont parallèles aux électrodes. Le champ est constant et est donné par :

$$
E=\frac{V_{0}}{d}
$$

On en déduit alors la densité de charge surfacique ainsi que la force électrostatique par unité de surface s'exerçant sur le miroir :

$$
\begin{aligned}
& \sigma=\varepsilon_{0} \cdot E=\varepsilon_{0} \cdot \frac{V}{d} \\
& F=\frac{\varepsilon_{0} \cdot V_{0}^{2}}{2 d^{2}}
\end{aligned}
$$

Considérons une rotation de la structure d'une distance $d$, comme montré dans la figure 3 . on a alors, en considérant que l'angle de rotation $\theta$ est faible :

$$
d=d_{0} \cdot\left(1-\frac{r \cdot \sin \theta}{d_{0}}\right) \approx d_{0} \cdot\left(1-\frac{r \cdot \theta}{d_{0}}\right)
$$

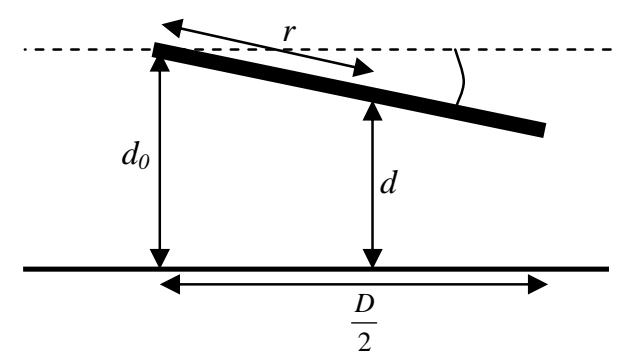

Figure 3. Modèle de la structure : prise en compte de la rotation. 
En intégrant l'équation (11) sur toute la surface $S$ de la plaque mobile, on obtient l'expression du moment électrostatique :

$$
M_{e}=\iint_{S} r \cdot F \cdot d S=\frac{\varepsilon_{0} \cdot V_{0}^{2} \cdot L}{2 \cdot \theta^{2}} \cdot\left[\ln \left(1-\frac{D \cdot \theta}{2 \cdot d_{0}}\right)+\frac{D \cdot \theta}{2 \cdot\left(d_{0}-\frac{D}{2 \cdot \theta}\right)}\right]
$$

L'expression générale donnant la tension d'actionnement en fonction de l'angle de rotation, pour des électrodes en configuration planes, s'obtient en égalisant les normes des moments électrostatique et mécanique. On obtient :

$$
V_{0}^{2}=\frac{4 k \cdot \theta^{3}}{\varepsilon_{0} \cdot L} \cdot\left[\ln \left(1-\frac{D \cdot \theta}{2 \cdot d_{0}}\right)+\frac{D \cdot \theta}{2 \cdot\left(d_{0}-\frac{D}{2 \cdot \theta}\right)}\right]^{-1}
$$

On obtient des caractéristiques statiques de la forme de la figure 4, pour un angle $\theta_{\max }$ égal à 2 degrés.

\section{Caractéristique statique du micro-miroir}

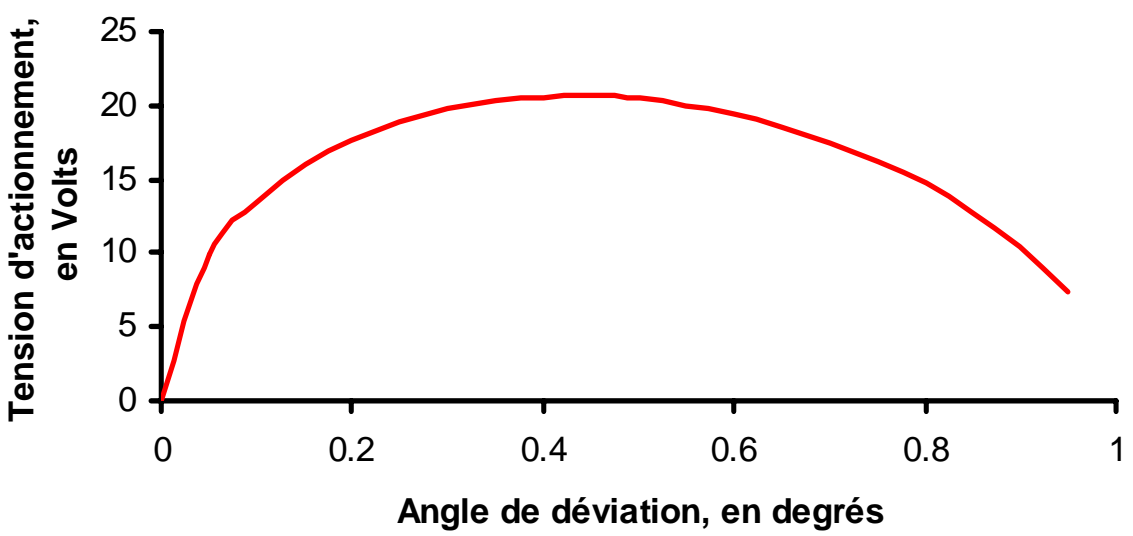

Figure 4. Caractéristique statique du micro-miroir considéré.

Comme la masse mobile, en l'occurrence le miroir, est suspendue par deux barres de torsion, cette dernière subit un couple mécanique s'opposant au couple électrostatique. L'angle de rotation du miroir dérive de l'égalisation des expressions de ces deux moments. Comme on le voit dans la courbe précédente, un cas spécial apparaît et est appelé tension de «pull-in ». 


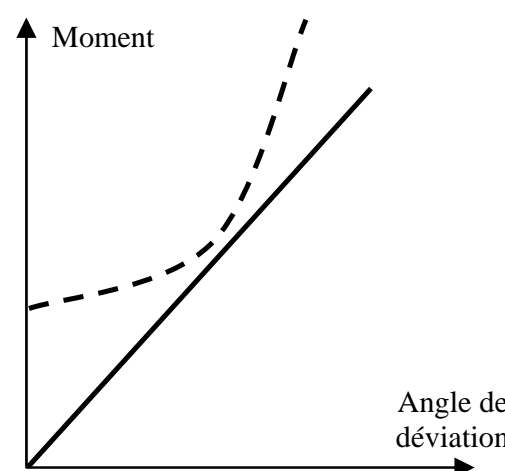

Cas 1.

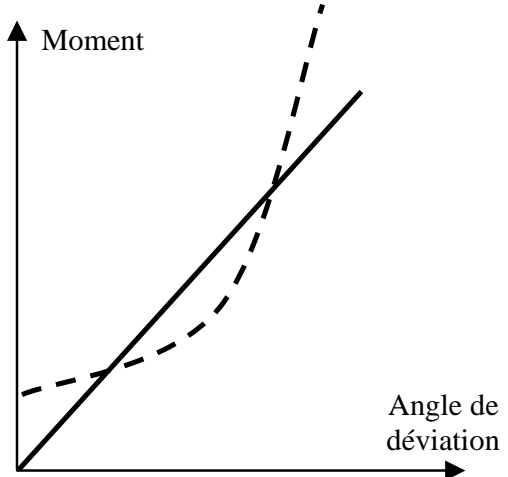

Cas 2.

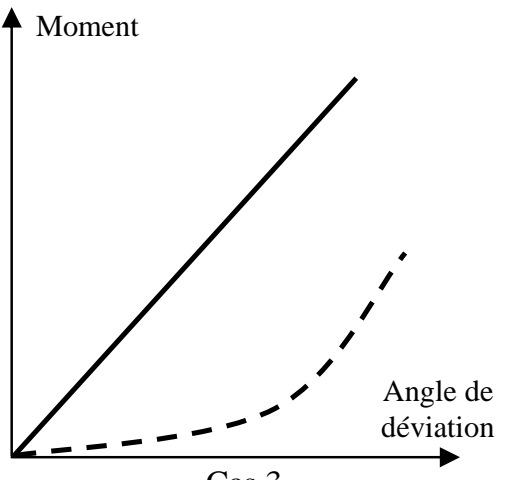

Cas 3.

Figure 5. Trois configurations possibles entre les moments électrotatique et mécanique.

Cette valeur de la tension correspond au moment où le couple électrostatique devient supérieur au couple mécanique, causant une attraction du miroir jusqu'au contact avec le premier obstacle sur sa trajectoire. Comparons graphiquement les positions relatives des moments électrostatiques (en pointillés) et mécaniques (en continu). Les trois cas possibles observables sont résumés dans la figure 5.

Le cas 1 représente des raideurs de ressorts faibles. Le moment mécanique est toujours inférieur au moment électrostatique. Aucun point d'équilibre ne peut être trouvé. Le cas 3 représente des raideurs de ressorts élevées. Le moment mécanique est toujours supérieur au moment électrostatique. Il n’y a donc jamais intersection entre les deux courbes, et donc pas de solution non plus.

Un cas spécial apparaît lorsque la tension de commande correspond au moment où le couple électrostatique devient supérieur au couple mécanique. A cette valeur, nommée par la suite $V_{\text {Pinn }}$ on observe une attraction du miroir jusqu'au contact avec le premier obstacle sur sa trajectoire. Cette valeur spéciale de la tension est appelée tension de «pull-in » [11]. L’angle $\theta_{\text {pin }}$ correspondant est donné par :

$\theta_{\text {pin }}=0.4404 \times \theta_{\max }$

La caractéristique statique du micro-miroir est alors modifiée. Un exemple est donné en figure 6.

\section{Caractéristique statique du micro-miroir}

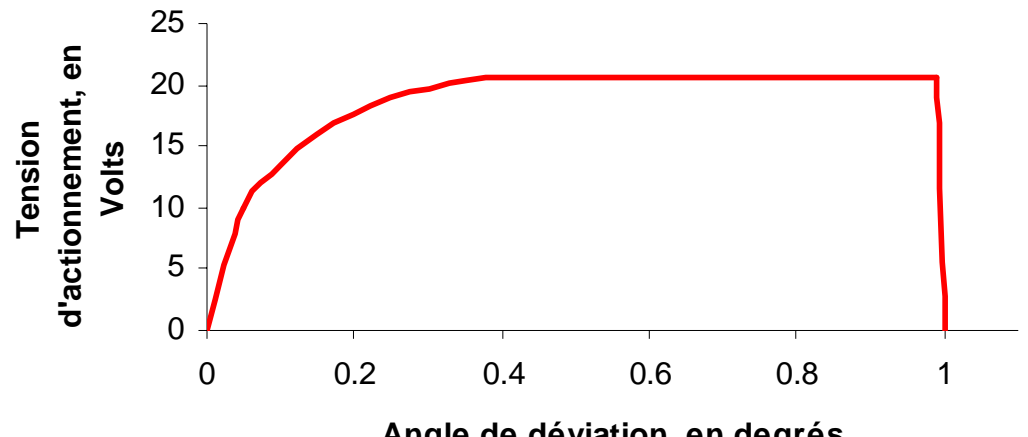

Figure 6. Caractéristique statique du micro-miroir considéré : Prise en compte des phénomènes de Pull-In. 


\subsection{Simulation aux éléments finis : préparation.}

Les dernières avancées dans le domaine des microsystèmes ont donné lieu à la création de nombreux outils de Conception Assistée par Ordinateur dédiés "Microsystèmes ». Pour valider nos résultats et permettre d'extraire de plus de nouveaux paramètres comme les temps de commutation, nous avons utilisés le logiciel COVENTORWARE développé par COVENTOR [7]. Il consiste à fabriquer un modèle 3D de la structure à partir de son layout et à effectuer des résolutions aux éléments finis afin d'extraire les effets mécaniques, capacitifs, thermiques, etc...

La première tâche à effectuer consiste à décrire les principales étapes de notre processus technologique. Parmi les nombreuses solutions proposées, nous avons choisi un process s'inspirant de celui utilisé dans le Multi Project Wafer MEMSOI II proposé par Tronic’s Microsystems [12-13]. Les principales étapes sont décrites dans les tableaux 1 et 2.

\begin{tabular}{|c|c|c|c|}
\hline Etape & Nature & Matériau & Epaisseur $(\mu \mathrm{m})$ \\
\hline 0 & Substrat de départ & Silicium & 50 \\
\hline 1 & Dépôt & Oxyde de Silicium & 0.4 \\
\hline 2 & Dépôt & Or & 2 \\
\hline 3 & Gravure & Or pour électrodes & 2 \\
\hline
\end{tabular}

Tableau 1. Etapes de réalisation des électrodes.

\begin{tabular}{|c|c|c|c|}
\hline Etape & Nature & Matériau & Epaisseur $(\mu m)$ \\
\hline 0 & Substrat de départ & Silicium & 50 \\
\hline 1 & Dépôt & Oxyde de Silicium & 0.4 \\
\hline 2 & Dépôt & Silicium & 15 \\
\hline 3 & Gravure & Or pour électrodes & 15 \\
\hline 4 & Gravure & Silicium face arrière & 50 \\
\hline 5 & Gravure & Libér. de la structure & \\
\hline
\end{tabular}

Tableau 2. Etapes de réalisation du miroir.

En combinant le fichier contenant la vue bi-dimensionnelle de la structure avec le processus technologique, on obtient une vue 3D de la structure, selon le processus décrit en figure 7.
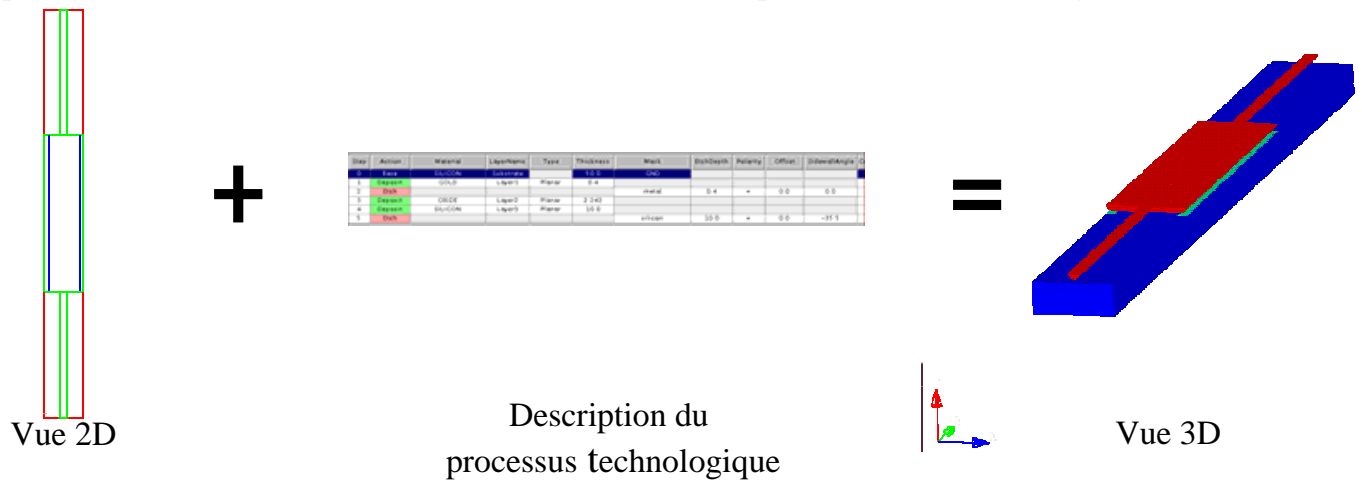

Figure 7. Construction du modèle 3D. 
Le modèle 3D étant construit, définissons maintenant le maillage de la structure, ainsi que les surfaces intéressantes. Deux solutions sont disponibles :

- Maillage "libre» : le maillage dit libre génère automatiquement les nœuds et les éléments sur les surfaces et les volume du modèle. Plusieurs motifs de maillage sont proposés : triangulaire (ne permet alors qu'un maillage surfacique), tétraédrique ou parallélépipédique; l'utilisateur a de plus la possibilité de contrôler les dimensions de la figure élémentaire de maillage. De ce fait, des structures aux formes complexes peuvent être très facilement modélisées.

- Maillage «en nappes » : contrairement au maillage libre, le maillage en nappe est régulier, effectué avec des éléments dont toutes les dimensions sont contrôlable par l'utilisateur. Ce type de maillage ne peut être appliqué qu'à des surfaces ou des volumes, pas à des courbes. Dans le cas de maillage de surface, ces dernières doivent posséder trois ou quatre faces. Pour des volumes, ces derniers doivent posséder cinq ou six faces, sans trous.

Le nombre de nœuds de calcul est fonction de l'utilité et du type d'analyse que l'on veut conduire. En effet, une pièce mobile, dans laquelle on veut connaître les contraintes crées par l'application d'un potentiel ou le déplacement, sera maillée avec des éléments volumiques comprenant un nombre de points de calcul compris entre 20 et 27 nœuds par structure élémentaire de maillage. Par opposition, Un élément fixe ne subira que des contraintes surfaciques: un maillage à l'aide d'éléments simples comprenant 4 nœuds de calcul sera suffisant.

Une fois la structure maillée, il reste à déterminer les propriétés physiques des matériaux utilisés, et fixer d'autre part les conditions aux limites de la structure.

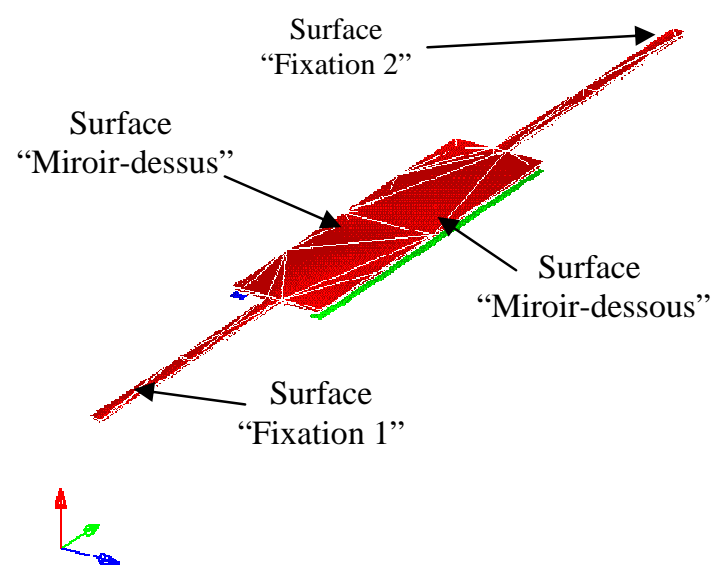

Figure 8. Vue du miroir maillé - Conventions prises.

Considérons tout d'abord les propriétés physiques des matériaux utilisés. Dans notre cas, seules la partie mobile et les électrodes nécessitent un maillage : on cherche dans un premier temps à connaître les contraintes dans les barres de torsion, ainsi que les déplacements en fonction des tensions appliquées. Il est donc important de connaître les propriétés de l'or ainsi que du silicium. COVENTOR possède pour cela une base de données, la Material Property Database, contenant un ensemble de valeurs numériques pour les différentes constantes physiques caractérisant les matériaux. Ceux utilisés sont décrits dans l'annexe de ce papier. 
Considérons maintenant les conditions aux limites que nous allons imposer au miroir. Ces dernières doivent rendre compte de son comportement réel.

Dans la figure précédente, on remarque que le cadre supportant les fixations des barres de torsion n'est pas représenté. Or, il faut bien imposer à ces dernières une absence de rotation suivant les trois dimensions $x, y$ et $z$. COVENTOR utilise pour cela la notion de patch : chaque élément de la structure est décomposé en surfaces. Par exemple, un cube possédant six faces est décomposé en six patchs. De ce fait, on appelle les deux extrémités libres du miroir « Fixation 1 » et « Fixation 2 », et on supprime tout mouvement à ces dernières.

\subsection{Simulation aux éléments finis : résultats.}

Le but des simulations que nous effectuons dans cette partie est de connaître le comportement mécanique et électrostatique du miroir. Nous utiliserons pour cela le module CoSolve-EM, permettant d'effectuer à la fois les deux types de calcul, grâce aux modules MemMech et MemCap de COVENTOR.

Présentons un peu CoSolve-EM. Ce solveur couplé permettant l'application des conditions aux limites combine le calcul des solutions mécaniques et capacitives. Dans un processus itératif, les solveurs MemCap et MemMech calculent les solutions en se basant sur les résultats précédents. La limite de convergence contrôle le nombre d'étapes requises permettant d'atteindre une solution stable. Les figures suivantes montrent des captures d'écran du miroir simulé.

\section{Comparaison des modèles analytiques et simulés}

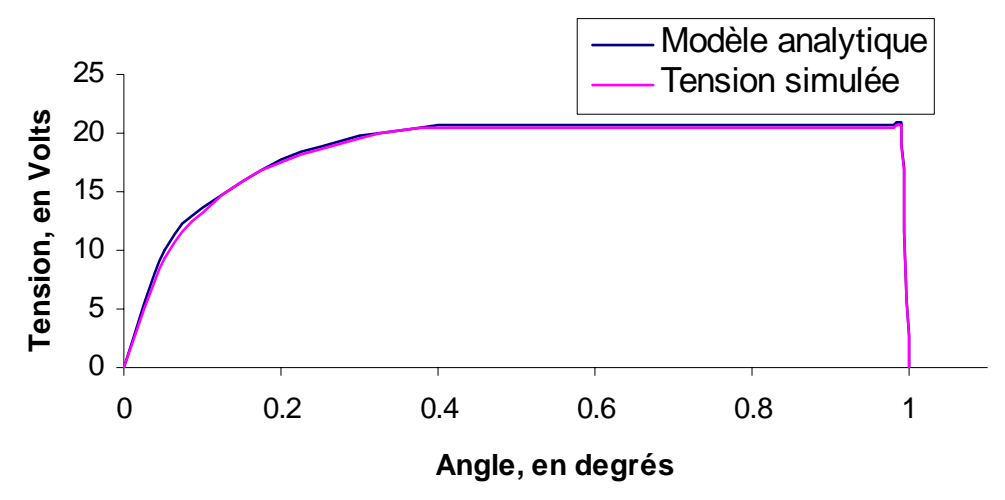

Figure 9. Comparaison des tensions d’actionnement simulées et calculées. 


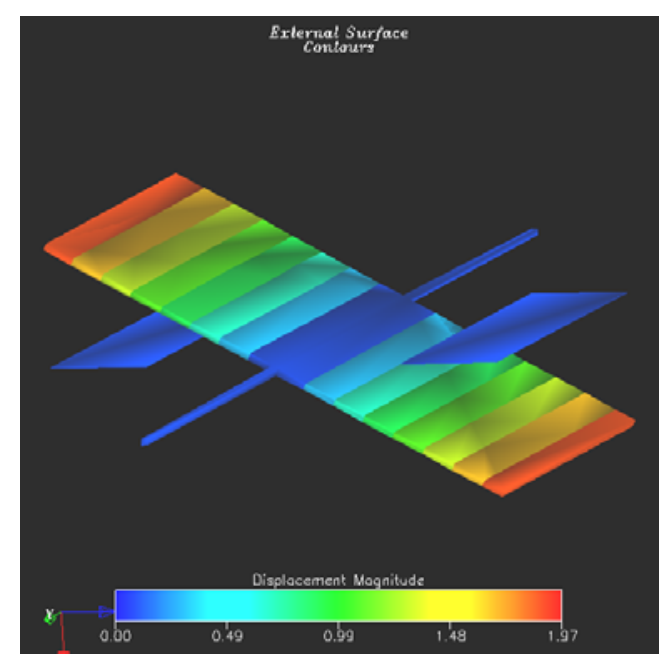

Figure 10. Déplacement du miroir sous application d'une tension de 15V.

On remarque une bonne concordance des résultats provenant de la simulation et du modèle numérique. Les écarts repérés proviennent à priori de la prise en compte de la flexion de la structure par Coventor, et la non-prise en compte dans le modèle numérique. Ainsi, le miroir se rapproche plus des électrodes de commande, et les tensions nécessaires pour faire basculer les miroirs diminuent.

\section{SIMULATION SOUS SABER}

\subsection{Généralités.}

Une fois les principales simulations effectuées en statique, permettant d'extraire les tensions de commande en fonction de l'angle de basculement, notre but est d'évaluer le temps de commutation du micro-miroir. Mais la connaissance de ce paramètre implique une simulation dynamique de la structure, c'est à dire la prise en compte en instantané de l'équation du mouvement du micro-miroir. Cette dernière faisant appel à de multiples domaines énergétiques, la détermination du temps de commutation par simulation tri-dimensionnelle est trop consommatrice en temps de calcul.

Une solution permettant de réduire la complexité des modèles considérés est de générer des modèles électriques dits à ordres réduits, insérables par la suite dans des simulateurs électriques tel que SABER. Cette méthode est basée sur une approche énergétique dans laquelle chaque domaine énergétique est modélisé séparément, puis ré-assemblé afin de décrire le comportement global du système.

\subsection{Modèle électrique du micro-miroir.}

Si l'on considère maintenant le cas de notre micro-miroir, ce dernier peut être décomposé en plusieurs sous-ensembles, chacun représentant un modèle énergétique précis, comme le montre le schéma de la figure ci-dessous : 


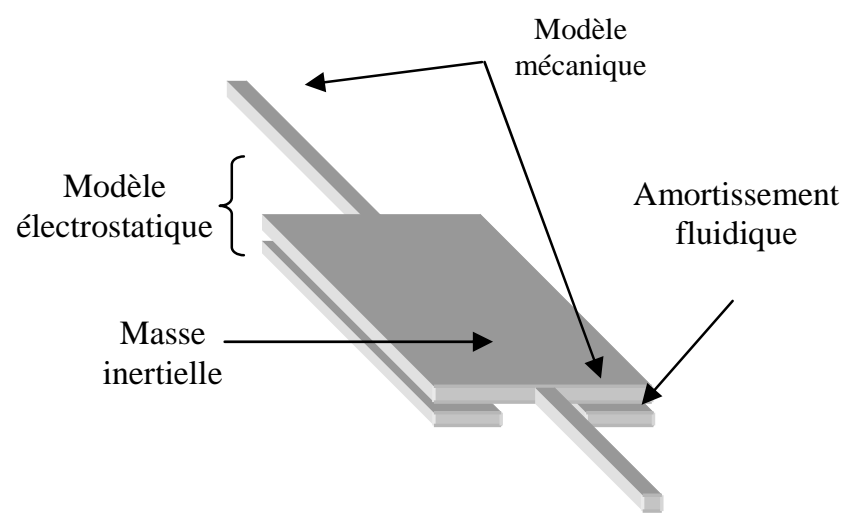

Figure 11. Modèles énergétiques considérés.

De ce fait, nous avons donc à modéliser tour à tour, comme brique élémentaire :

- Un modèle "électrostatique »: ce dernier représentera l'effet capacitif dans la structure. Pour différentes valeurs de l'angle de rotation, et suivant les différents degrés de liberté fixés, une matrice représentative des différentes capacités calculées est extraite. Un polynôme, de degré supérieur à 4, est alors calculé, approchant au mieux les résultats obtenus.

- Un modèle « mécanique » : comme dans le cas précédent, les forces résultant de la rotation de la barre de torsion sont évaluées pour différentes valeurs d'angles, pour les différents degrés de liberté considérés. Les résultats sont ensuite approximés par un polynôme de degré supérieur à 4 .

- Une masse inertielle : la surface du miroir est assimilée à une masse en mouvement, caractérisée essentiellement par son moment d'inertie.

En considérant que le miroir n'a la possibilité que de tourner d'un angle $\theta$ autour de son axe de rotation, ou de fléchir suivant une direction perpendiculaire à la surface d'une amplitude $z$, ce système à deux degrés de liberté peut être décrit, en utilisant le principe fondamental de la dynamique appliqué au miroir, par :

$$
\begin{aligned}
& M \ddot{z}=-k^{\prime} z-F_{z} \cdot \dot{z}+\frac{V^{2}}{2} \cdot \frac{d C}{d z} \\
& I \ddot{\theta}=-G \theta-F_{\theta} \cdot \dot{\theta}+\frac{V^{2}}{2} \cdot \frac{d C}{d \theta}
\end{aligned}
$$

dans laquelle $M$ et $I$ sont respectivement la masse et le moment d'inertie, $k$ ' et $G$ sont les raideurs en flexion et en torsion des barres de torsion, $F_{z}$ et $F_{\theta}$ les coefficients d'amortissement, $V$ la tension appliquée et $C$ la capacité. La figure 12 montre le schéma du modèle du micro-miroir implémenté sous SABER. 


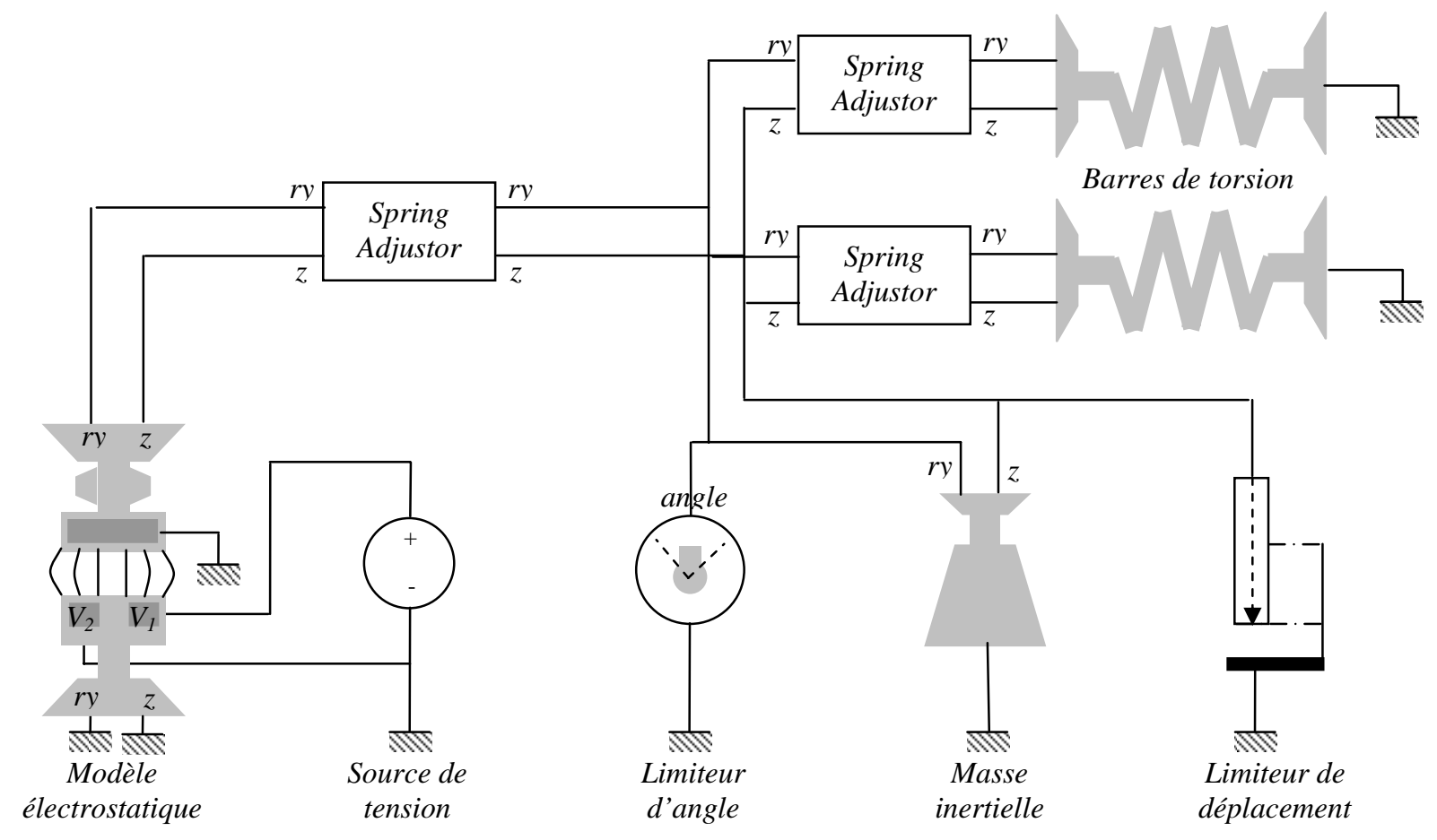

Figure 12. Schéma du micro-miroir implémenté sous SABER.

\subsection{Dimensionnement du convertisseur.}

La dernière étape de notre travail de modélisation consiste maintenant à simuler le convertisseur qui alimentera notre micro-miroir. Dans le cas étudié, on considère avoir en entrée une tension continue de $3 \mathrm{~V}$, correspondant à l'ordre de grandeur de la tension disponible dans tout circuit microélectronique. Le convertisseur devra délivrer en sortie une tension de 30V, nécessaire à l'actionnement du micromiroir. Pour élever la tension, nous avons décidé d'utiliser un hacheur parallèle ou convertisseur de type Boost, dont la structure est présentée en figure 13.

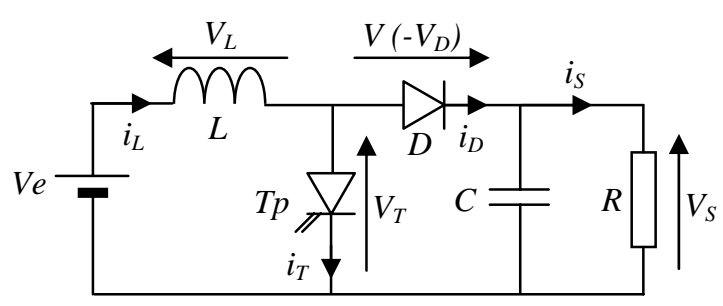

Figure 13. Structure Boost simulée.

Son principe de fonctionnement est le suivant : l'interrupteur $T p$ est fermé pendant un temps $\alpha T$. L'énergie est stockée dans l'inductance $L$, la diode $D$ est bloquée. Le blocage de $T p$ entraîne la décharge de l'inductance dans la charge $R$. Cette décharge n'est possible que pour $V s>V e$. 
La condition $V l_{\text {moyen }}=0$ en régime permanent impose $V e=V t_{\text {moyen }}$, donc :

$V_{S}=\frac{1}{1-\alpha} \cdot V_{e} \quad$ et $\quad y=\frac{1}{1-\alpha}$

Pour $\alpha$ compris entre 0 et 1 , la tension de sortie est toujours supérieure à $V e$, le montage est alors élévateur de tension (survolteur).

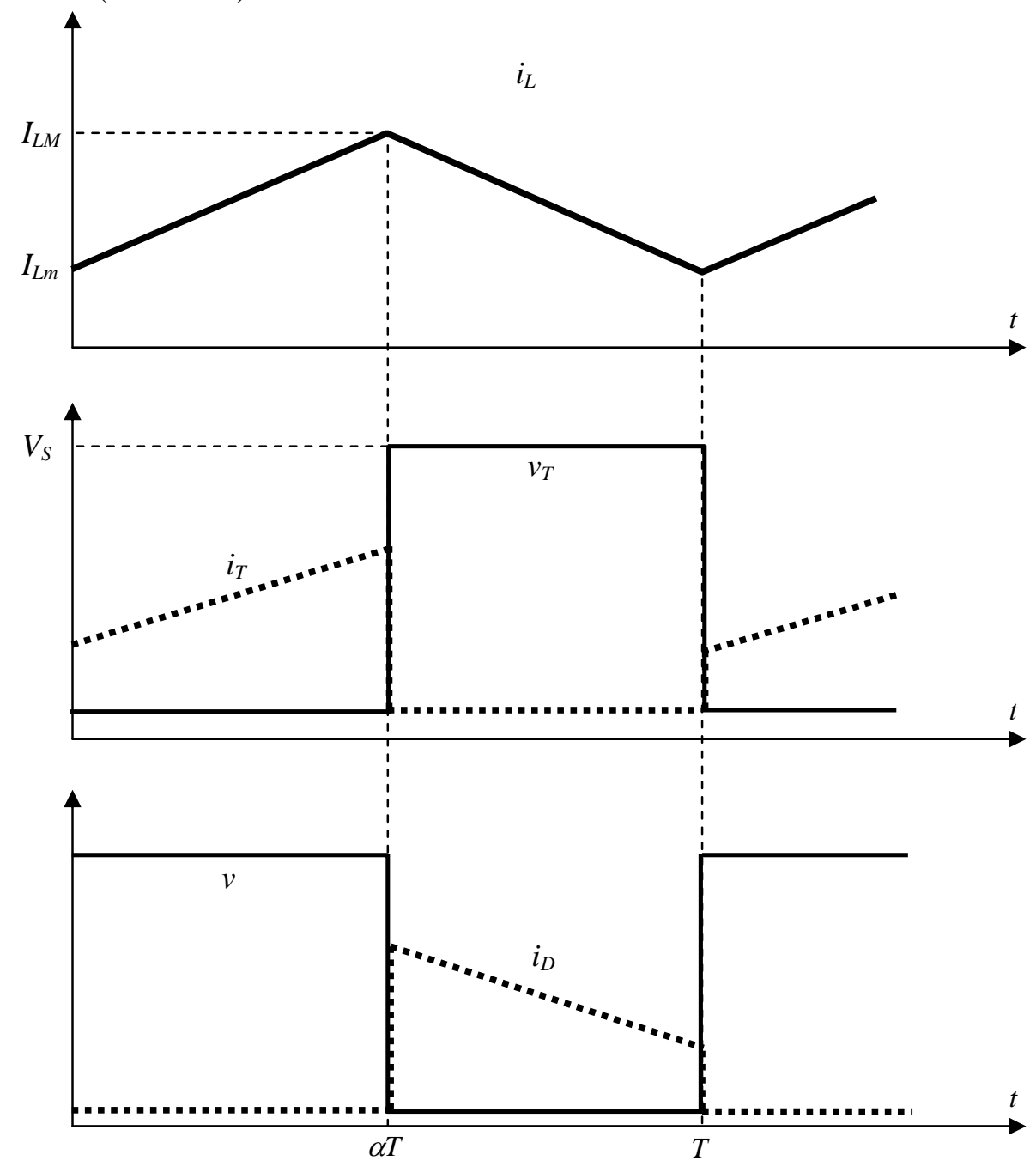

Figure 14. Formes d'ondes.

Les hypothèses de fonctionnement sont: $\mathrm{V}_{\text {in }}=3 \mathrm{~V}, \mathrm{~V}_{\text {out }}=30 \mathrm{~V}, \mathrm{I}_{\text {in }}=1 \mathrm{~A}$. Le premier paramètre à évaluer est le rapport cyclique $\alpha$, défini par :

$$
V_{S}=\frac{1}{1-\alpha} \cdot V_{e} \quad \Leftrightarrow \quad \alpha=1-\frac{V_{e}}{V_{S}}
$$

Comme on impose dans les hypothèses que les tensions d'entrée $V_{e}$ et de sortie $V_{S}$ doivent respectivement être égales à $3 \mathrm{~V}$ et $30 \mathrm{~V}$, on en déduit que le rapport cyclique $\alpha$ doit être égal à 0.9 . De ce fait, le courant de sortie $I_{S}$, donné par (9), vaut $0.03 \mathrm{~A}$ 


$$
I_{S}=(1-\alpha) \cdot I_{L}
$$

Connaissant maintenant les courants et tensions de sortie, on en déduit la valeur de la résistance de charge $R$ :

$$
R=\frac{V_{S}}{I_{S}}
$$

Les tensions et courants de sortie étant respectivement égaux à $30 \mathrm{~V}$ et $0.03 \mathrm{~A}$, on en déduit que la résistance de charge devra être de $10 \Omega$. le point suivant à examiner est la valeur de la capacité. Si l'on suppose que le courant de sortie est régulé, et égal à $0.03 \mathrm{~A}$, et si la fréquence de commutation du transistor est de $10 \mathrm{kHz}$, on en déduit la valeur de la capacité :

$$
C=i \cdot \frac{d t}{d V} \quad \Leftrightarrow \quad C=i \cdot \frac{V_{S}}{\Delta V_{S}} \cdot \frac{T}{2} \cdot \frac{1}{V_{S}}
$$

On fixe $\frac{\Delta V_{S}}{V_{S}}$ égal à $1 \%$, et un temps égal à une demi-période. De ce fait, la capacité a pour valeur 1.5nF. Enfin, reste à évaluer la valeur de l'inductance. En effet, on l'utilise dans notre cas pour lisser le courant de sortie et stocker une partie du courant durant une partie de la période. La tension aux bornes de l'inductance est donnée par :

$$
V_{L}=L \cdot \frac{d I}{d t} \quad \Leftrightarrow \quad L=V_{L} \cdot \frac{d t}{d I}
$$

Quand le transistor est en mode de conduction, la tension aux bornes de l'inductance est égale à la tension d'entrée, soit :

$$
V_{L} \approx V_{e} \approx L \cdot \frac{d I}{d t}
$$

Comme on fixe $\Delta \mathrm{I}=10 \%$ de $I_{\text {avg}}$, on en déduit que l'inductance devra être égale à $1.5 \mathrm{nH}$. Après des simulations conduites sous SABER, on en déduit les valeurs précises de $L$ et $C$, dans le but de diminuer et d'affiner les taux d'ondulation des tensions et courants. On obtient, pour un ripple de $1 \%, \mathrm{~L}=2.5 \mathrm{nH}$, et $\mathrm{C}=1.5 \mathrm{nF}$.

\subsection{Résultats.}

L'alimentation du micro-miroir étant conçue, nous avons associé sous SABER les modèles électriques de l'alimentation et du micro-miroir. Le tableau 3 résume les résultats obtenus, pour différents signaux appliqués en entrée. 


\begin{tabular}{|c|c|c|c|c|}
\hline Signal & $\begin{array}{c}\text { Tension appliquée, } \\
\text { en V. }\end{array}$ & Période, en ms. & $\begin{array}{c}\text { Tension de } \\
\text { basculement, en V. }\end{array}$ & $\begin{array}{c}\text { Temps de } \\
\text { basculement, en ms }\end{array}$ \\
\hline Impulsion & 30 & 0.001 & 19 & 10 \\
\hline Impulsion & 25 & 0.001 & 20 & 20 \\
\hline Carré & 30 & 0.1 & 20 & 28 \\
\hline Carré & 25 & 0.01 & 20 & 31 \\
\hline
\end{tabular}

Tableau 3. Résultats obtenus après simulation sous SABER

On remarque une bonne concordance entre les tensions calculées avec les trois modèles. Les approximations faites lors de la décomposition du micro-miroir en sous-ensembles semblent cohérentes.

\section{CONCLUSION}

Un micro-miroir a été simulé dans cet article en utilisant les logiciel COVENTORWARE et SABER. Dans un premier temps, les paramètres clefs du microsystème ont été extraits grâce à un modèle analytique, puis validés par le logiciel aux éléments finis, Un modèle électrique du miroir a été ensuite extrait grâce à COVENTOR, puis introduit sous SABER, afin d'effectuer la simulation du miroir avec son alimentation, et d'extraire un modèle dynamique du micro-miroir.

\section{REMERCIEMENTS}

Ce projet est soutenu par les activités de recherche présentes à l'INSA et dans son environnement de recherche proche (LAAS-CNRS). Il est réalisé grâce aux moyens informatiques mis à notre disposition à l’Atelier Inter-universitaire de Micro-Électronique de Toulouse (AIME).

\section{REFERENCES}

[1]D. Estève, J. Simonne, « Microsystèmes », Techniques de l'Ingénieur, n ${ }^{0}$ E 2305, pp.1-17, 1998.

[2]R.H.K., "Optical Networks: global competition for new LH DWDM buildings in Fierce", Telecommunications Industry Analysis, pp.1-10, 2002.

[3] A. Permuy, “ Capteurs microélectroniques », Techniques de l’Ingénieur, n ${ }^{0}$ E 2315, pp.1-17, 1993.

[4] K. Grenier, D. Dubuc, L. Rabbia, A. Takacs, P. Pons, T. Parra, P. Caudriller, H.Aubert, J. Graffeuil, O. Pascal, P. Combes, H. Baudrand, R. Plana, «MEMS devices for the future wireless applications », 4th IEEE International Caracas Conference on Devices, Circuits and Systems (ICCDCS'2002), pp.D031.1-D031.8, 2002. 
[5] J-Y. Fourniols, A. Martinez, C. Vieu, B. Pradin, F. Voillot, J-P. Ulmet, P. Gérard, J-L. Noullet, B. Estibals, «Microsystems-Microtechnologies : design, test and characterisation in educational context at INSA Toulouse”, Procedings of CETSIS-EEA, Clermont-Ferrand, 2001.

[6]B. Estibals, J-Y. Fourniols, J-L. Noullet, A. Martinez, "MEMS Design, realisation and characterisation in an educational context", IEE Engineering and Education Science, vol. 11, n ${ }^{\circ}$ 6, 2001.

[7] Site Internet : www.coventor.com

[8] Site Internet : www.avanti.com

[9]S. Renard, B. Estibals, V. Gaff, "Optical MEMS Components become Key Players in Optical Networks”, Europhotonics, n ${ }^{0}$ April/May01, pp. 33-35, 2001.

[10]W. Young, "Roark's Formulas for Stress and Strain", $6^{\text {th }}$ edition, McGraw-Hill Book Company, 1989.

[11]Y. Neminrovsky, O. Bochobza-Degani, "A Methodology and Model for the Pull-In Parameters of Electrostatic Actuators”, IEEE Journal Of Microelectromechanical Systems, vol. 10, n ${ }^{0}$ 4, pp. 601614, 2001.

[12]S. Renard, "Industrial MEMS on SOI”, Journal of Micromech. Microeng., vol. 10, pp. 245-249, 2000.

[13]Site Internet : www.tronics-mst.com

\section{ANNEXES}

- Dimensions utilisées dans l'exemple.

\begin{tabular}{|l|c|c|c|}
\cline { 2 - 4 } \multicolumn{1}{c|}{} & $L$ & $l$ & $e$ \\
\hline Miroir $(\mu \mathrm{m})$ & 600 & 400 & 15 \\
\hline Barres de torsion $(\mu \mathrm{m})$ & 600 & 10 & 15 \\
\hline Electrodes $(\mu \mathrm{m})$ & 600 & 110 & 90 \\
\hline
\end{tabular}

Tableau 4. Dimensions utilisées dans l’exemple.

$L=$ Longueur, $l=$ largeur, $e=$ épaisseur sauf pour les électrodes, où $e$ caractérise l'espacement entre elles.

- Grandeurs physiques utilisées dans l'exemple pour caractériser les matériaux.

\begin{tabular}{|l|c|c|c|}
\hline Grandeurs physique & Unité & Silicium & Or \\
\hline Densité & $\mathrm{kg} / \mu \mathrm{m}^{3}$ & $2.510^{-15}$ & $1.9310^{-14}$ \\
Conductivité thermique & $\mathrm{pW} / \mu \mathrm{mK}$ & $1.4810^{8}$ & $2.9710^{8}$ \\
Chaleur spécifique & $\mathrm{pJ} / \mathrm{kgK}$ & $7.1210^{14}$ & $1.2910^{14}$ \\
Conductivité électrique & $\mathrm{pS} / \mu \mathrm{m}$ & & $4.410^{13}$ \\
\hline
\end{tabular}

Tableau 5. Paramètres physiques utilisés et valeurs numériques. 